\title{
Plasma Fatty Acid Composition, Estimated Desaturase Activities, and Intakes of Energy and Nutrient in Japanese Men with Abdominal Obesity or Metabolic Syndrome
}

\author{
Aiko KaWAShima ${ }^{1}$, Shiori Sugawara ${ }^{1}$, Misako OKITA ${ }^{1}$, Takemi AKAHANE ${ }^{2}$, Kennichi FuKUI ${ }^{2}$, \\ Maiko HASHIUCHI ${ }^{2}$, Chieko KATAOKA ${ }^{2}$ and Ikuyo TsUKAMOTO ${ }^{1}$ \\ ${ }^{1}$ Department of Food Science and Nutrition, Nara Women's University, Nara 630-8506, Japan \\ ${ }^{2}$ Nara Health Promotion Center, Tawaramoto-cho, Nara 630-8506, Japan
}

(Received March 9, 2009)

\begin{abstract}
Summary To examine predictive factors for abdominal obesity or metabolic syndrome, we investigated the association of plasma fatty acid composition, estimated desaturase activity, and nutrient intakes, with abdominal obesity or metabolic syndrome in Japanese males. Clinical characteristics, the fatty acid composition of plasma cholesteryl esters, and energy and nutrient intakes were analyzed in 3 groups: metabolic syndrome (MS, $n=24$ ), abdominal obesity (OB, $n=43)$, and control $(n=27)$. The estimated desaturase activities were calculated by the ratio of 16:1n-7/16:0, 18:3n-6/18:2n-6, and 20:4n-6/20:3n-6 in plasma cholesteryl esters as surrogates of the measure of the delta 9, delta 6, delta 5 desaturase (D916D, D6D and D5D) activities, respectively. Plasma fatty acid composition did not differ significantly between the OB group and the control group. The MS group had higher levels of palmitoleic, oleic, and $\gamma$-linolenic acids, but a lower level of linoleic acid than the control. Stronger D6D activity and weaker D5D activity were observed in the OB group. A higher level of D9-16D activity as well as a higher level of D6D activity and a lower level of D5D activity was observed in the MS group. A logistic regression analysis showed that the low D5D activity and high D9-16D activity were predictive of the development of abdominal obesity from controls (odds ratio $=0.39, p<0.05$ ) and metabolic syndrome from abdominal obesity (odds ratio $=2.44, p<0.05$ ), respectively. In the multiple linear regression analysis, D5D activity positively correlated with the intake of eicosapentaenoic acid (EPA). In conclusion, the estimated D5D activity was a predictive factor for abdominal obesity and the estimated D9-16D activity was a predictive factor for developing metabolic syndrome from abdominal obesity in Japanese male subjects. Dietary intake of EPA would play an important role in preventing abdominal obesity and the development of metabolic syndrome.

Key Words delta 5 desaturase, delta 9 desaturase, dietary intake of eicosapentaenoic acid (EPA), abdominal obesity, metabolic syndrome
\end{abstract}

Metabolic syndrome is a metabolic disorder that results from abdominal obesity. The syndrome includes insulin resistance, hypertension and dyslipidemia (1), and is a risk factor for cardiovascular disease and type 2 diabetes (2). A high intake of fat, particularly fat rich in saturated fatty acids (SFAs), influenced obesity, insulin resistance and the progression of metabolic syndrome $(3,4)$. A high intake of carbohydrate also induced insulin resistance, increased serum triacylglycerol concentrations and decreased serum HDL cholesterol levels (5). Thus, dietary nutrient intake plays an important role in the development of obesity and metabolic syndrome.

The fatty acid composition of cholesteryl esters (CEs) in serum mirrors to a certain extent the dietary fatty acid composition and also reflects endogenous fatty acid metabolism (6-9). Fatty acid composition is used as an indicator of disease risk, because its alteration has been

E-mail: faa_kawashima@cc.nara-wu.ac.jp related to metabolic disease and cardiovascular disease $(10,11)$. Low concentrations of linoleic acid (LA, 18:2n-6) and high concentrations of palmitic (16:0), palmitoleic (16:1n-7) and dihomo- $\gamma$-linolenic (20:3n-6) acids in plasma lipid esters have been reported to be associated with metabolic syndrome $(12,13)$.

Desaturases are involved in the endogenous synthesis of polyunsaturated fatty acids (PUFAs). The delta 9, 6, and 5 desaturases (D9D, D6D, D5D) introduce a double bond at specific positions on long-chain fatty acids. D9D synthesizes monounsaturated fatty acids (MUFA), palmitoleic (16:1n-7) and oleic (18:1n-9) acids from palmitic (16:0) and stearic (18:0) acids, respectively. D5D and D6D catalyze the synthesis of long-chain $n-6$ and $n-3$ PUFAs. In human studies, the estimated desaturase activities are generally used, since it is not possible to directly measure desaturase activities in humans (9, 13-15). The estimated activities of D9D, D6D and D5D calculated by the plasma ratio of $16: 1 n-7 / 16: 0$, $18: 3 n-6 / 18: 2 n-6$, and 20:4n-6/20:3n-6, respectively, 
can be used as surrogates of the measure of the true desaturase activity (9). The validity of the use of the plasma fatty acid ratio as an in vivo surrogate was shown in several mouse strains and human subjects (16). The associations between estimated desaturase activities and insulin action in humans accord well with results from animal studies where true enzyme activities have been measured (9). High levels of the estimated D9D activity were observed in patients with diabetes, atherosclerosis, obesity and metabolic syndrome $(13,14)$. D6D activity was also increased in these conditions, while $\mathrm{D} 5 \mathrm{D}$ activity was decreased (13-15). An alteration in the estimated D5D activity was shown to be predictive of the occurrence of metabolic syndrome $20 \mathrm{y}$ later in middle-aged men (14). However, the differences in fatty acid composition and desaturase activities between abdominal obesity and metabolic syndrome are still unknown. Predictive factors for the development of abdominal obesity from nonobesity and the development of metabolic syndrome from abdominal obesity have not been reported either.

In this study, to examine predictive factors for abdominal obesity and metabolic syndrome, we investigated the association of plasma fatty acid composition, estimated desaturase activity, and dietary nutrient intakes with abdominal obesity and metabolic syndrome in Japanese males.

\section{SUBJECTS AND METHODS}

Subjects. This study was conducted on people who underwent a health check-up at Nara Health Promotion Center. Of 187 males invited to participate, 119 provided informed consent. Participants receiving treatment for diabetes, hypertension and dyslipidemia were excluded. Finally, data from 94 participants were analyzed. Subjects were classified into 3 groups: metabolic syndrome (MS), abdominal obesity (OB) and control. According to the definition of the Japanese Committee for the Diagnostic Criteria of Metabolic Syndrome (17), MS was defined based on abdominal obesity (waist circumference $\geqq 85 \mathrm{~cm}$ ) plus 2 or more of the following 3 criteria: dyslipidemia (triglyceride $\geqq 150 \mathrm{mg} / \mathrm{dL}$ and/ or high density lipoprotein-cholesterol (HDL-c) $<40 \mathrm{mg} / \mathrm{dL}$ ), elevated blood pressure (systolic blood pressure $\geqq 130 \mathrm{mmHg}$, and/or diastolic blood pressure $\geqq 85 \mathrm{mmHg}$ ), and impaired glucose tolerance (fasting glucose $\geqq 110 \mathrm{mg} / \mathrm{dL}$ ). OB was defined by the presence of abdominal obesity plus one or none of the 3 criteria. The control group had no abdominal obesity, dyslipidemia, diabetes or elevated blood pressure. The number of subjects in the MS, OB, and control groups was $27(50.4 \pm 6.1 \mathrm{y}), 43(51.0 \pm 5.6 \mathrm{y})$ and 24 $(49.3 \pm 6.6 \mathrm{y})$, respectively. This study was designed in accordance with the principles of the Declaration of Helsinki of the World Medical Association and was approved by the Ethics Committee of Nara Health Promotion Center and Nara Women's University.

Clinical data. Body mass index (BMI) was calculated as body weight $(\mathrm{kg})$ divided by squared body height $(\mathrm{m})$. Waist circumference was measured at the level of the umbilicus in the standing position.

All serum and plasma samples were obtained in the fasting state. Routine clinical parameters such as systolic blood pressure, diastolic blood pressure, serum total cholesterol, serum TG, low-density lipoprotein (LDL)-cholesterol, HDL-cholesterol, fasting blood glucose, fasting insulin and $\mathrm{HbA} 1 \mathrm{c}$ were measured. The insulin resistance index was calculated using homeostasis model assessment (HOMA-IR) (18). The serum concentrations of adiponectin and leptin were determined by ELISA (Otsuka Pharmaceutical Co., Ltd., Tokyo, Japan).

Fatty acid analysis. Plasma was separated by the centrifugation of fasting blood samples containing EDTA-2Na at $1,600 \times g$ for $15 \mathrm{~min}$ at $4^{\circ} \mathrm{C}$ and stored at $-80^{\circ} \mathrm{C}$. Total lipid was extracted from plasma by the method of Bligh and Dyer (19). CEs were separated by thin-layer chromatography using silica gel plates (Silica Gel 60; Merck, Darmstadt, Germany) with a solvent system of petroleum ether : ethylether : acetic acid ( $80: 20: 1, \mathrm{v} / \mathrm{v} / \mathrm{v})$. The spot corresponding to CEs was scraped from the plate and transmethylated with $2 \mathrm{~mL}$ of acetyl chloride : methanol $(5: 50, \mathrm{v} / \mathrm{v})$ at $90^{\circ} \mathrm{C}$ for $2 \mathrm{~h}$. Heptadecanoic acid (17:0) was used as an internal standard. Fatty-acid methyl esters were analyzed by gas-liquid chromatography (Model GC-2014; Shimadzu, Kyoto, Japan) equipped with a $25 \mathrm{~m} \times 0.5 \mathrm{~mm}$ capillary column (HR-SS-10, Shinwa Chemical Industries Ltd., Kyoto, Japan) as described (20).

Estimation of desaturase activity and lipogenic index. The estimated desaturase activity was calculated by the ratio of the fatty acids in plasma CEs as follows. D9-16D activity $=[16: 1 n-7 / 16: 0], \mathrm{D} 9-18 \mathrm{D}$ activity $=[18: 1 n-9 /$ 18:0], D6D activity=[18:3n-6/18:2n-6] and D5D activity $=[20: 4 n-6 / 20: 3 n-6]$. The ratio of palmitic acid (16:0) to linoleic acid (18:2n-6) in plasma CEs was used as a de novo lipogenic index (21).

Estimation of energy and nutrient intakes. Energy and nutrient intakes were assessed by using the food-frequency questionnaire (FFQ) designed to estimate food items in 29 food groups as described (22). In the FFQ, subjects were questioned on how often they consumed individual food groups during breakfast, lunch and dinner, as well as representative portion sizes (small, medium, and large). "Small" is half of "medium," and "large" is one and a half times the size of "medium." Daily nutrient intake was calculated by multiplying the frequency of consumption of each food by the nutrient content of the portion size and summing the products for all foods items in the FFQ. Daily intakes of nutrients were calculated with the use of the Fifth Revised and Enlarged Edition of the Standard Tables of Food Composition in Japan (23). The dietary intake of fatty acid was calculated according to a previous study (24).

Statistical analysis. Data are given as the mean \pm standard deviation (SD). Statistical analyses were done using SPSS v. 16.0 (SPSS Inc., Chicago, IL, USA). The normal distribution of variables was examined with Shapiro-Wilk's test, and non-normally distributed variables were log transformed. The group differences were 
Table 1. Clinical characteristics.

\begin{tabular}{|c|c|c|c|c|}
\hline & $\begin{array}{l}\text { Control } \\
(n=27)\end{array}$ & $\begin{array}{c}\mathrm{OB} \\
(n=43)\end{array}$ & $\begin{array}{c}\text { MS } \\
(n=24)\end{array}$ & ANOVA \\
\hline Age (y) & $49.3 \pm 6.6$ & $51.0 \pm 5.6$ & $50.4 \pm 6.1$ & \\
\hline BMI $\left(\mathrm{kg} / \mathrm{m}^{2}\right)$ & $22.2 \pm 1.6$ & $25.5 \pm 1.9^{*}$ & $26 \pm 2.6^{*}$ & $<0.001$ \\
\hline Waist circumference $(\mathrm{cm})$ & $81.0 \pm 3.4$ & $91.6 \pm 4.7^{*}$ & $93 \pm 5.6^{*}$ & $<0.001$ \\
\hline Systolic blood pressure $(\mathrm{mmHg})$ & $121 \pm 13$ & $125 \pm 13$ & $141 \pm 14^{* \#}$ & $<0.001$ \\
\hline Diastolic blood pressure (mmHg) & $77 \pm 9$ & $80 \pm 8$ & $90 \pm 9 * \#$ & $<0.001$ \\
\hline Total cholesterol $(\mathrm{mg} / \mathrm{dL})$ & $198 \pm 33$ & $213 \pm 30$ & $213 \pm 35$ & \\
\hline Triacylglycerol (mg/dL) & $96 \pm 46$ & $141 \pm 55^{*}$ & $201 \pm 74^{* \#}$ & $<0.001$ \\
\hline HDL cholesterol (mg/dL) & $62 \pm 14$ & $54 \pm 14^{*}$ & $51 \pm 12^{*}$ & $<0.001$ \\
\hline LDL cholesterol (mg/dL) & $118 \pm 26$ & $133 \pm 29$ & $125 \pm 34$ & \\
\hline Fasting blood glucose (mg/dL) & $94 \pm 6$ & $100 \pm 6^{*}$ & $116 \pm 21^{* \#}$ & $<0.001$ \\
\hline Fasting blood insulin $(\mu \mathrm{g} / \mathrm{mL})$ & $5.1 \pm 2.0$ & $8.5 \pm 4.1^{*}$ & $11.9 \pm 9.2^{*}$ & $<0.001$ \\
\hline $\mathrm{HbAlc}(\%)$ & $5.0 \pm 0.3$ & $5.3 \pm 0.3^{*}$ & $5.6 \pm 0.6^{* \#}$ & $<0.001$ \\
\hline HOMA-IR & $1.2 \pm 0.5$ & $2.1 \pm 1.0^{*}$ & $3.5 \pm 2.9^{*}$ & $<0.001$ \\
\hline Adiponectin (mg/mL) & $9.2 \pm 4.6$ & $7.2 \pm 3.4$ & $5.4 \pm 2.8^{*}$ & $<0.010$ \\
\hline Leptin (ng/mL) & $2.0 \pm 0.9$ & $4.7 \pm 2.9^{*}$ & $4.7 \pm 1.9^{*}$ & $<0.001$ \\
\hline
\end{tabular}

Mean \pm SD. ${ }^{*} p<0.05$; versus control group, ${ }^{*} p<0.05$; versus OB group. BMI, body mass index.

Table 2. Fatty acid compositions, estimated desaturase activities, and lipogenic index in plasma cholesteryl esters.

\begin{tabular}{|c|c|c|c|c|}
\hline & $\begin{array}{l}\text { Control } \\
(n=27)\end{array}$ & $\begin{array}{c}\mathrm{OB} \\
(n=43)\end{array}$ & $\begin{array}{c}\text { MS } \\
(n=24)\end{array}$ & ANOVA \\
\hline \multicolumn{5}{|l|}{ Fatty acids (mol\%) } \\
\hline Palmitic acid (16:0) & $12.24 \pm 1.10$ & $12.46 \pm 1.02$ & $12.62 \pm 1.35$ & \\
\hline Palmitoleic acid (16:1n-7) & $2.53 \pm 0.95$ & $2.74 \pm 1.14$ & $3.63 \pm 1.25^{* \#}$ & $<0.050$ \\
\hline Stearic acid (18:0) & $1.04 \pm 0.36$ & $0.98 \pm 0.19$ & $1.07 \pm 0.19$ & \\
\hline Oleic acid $(18: 1 n-9)$ & $17.66 \pm 2.62$ & $18.82 \pm 2.06$ & $19.59 \pm 2.19^{*}$ & $<0.050$ \\
\hline Linoleic acid (18:2n-6) & $51.84 \pm 4.92$ & $50.13 \pm 3.86$ & $47.98 \pm 4.81^{*}$ & $<0.010$ \\
\hline$\gamma$-Linolenic acid (18:3n-6) & $0.57 \pm 0.25$ & $0.84 \pm 0.50$ & $0.89 \pm 0.46^{*}$ & $<0.010$ \\
\hline$\alpha$-Linolenic acid (18:3n-3) & $0.74 \pm 0.38$ & $0.61 \pm 0.22$ & $0.76 \pm 0.96$ & \\
\hline Dihomo- $\gamma$-linolenic acid (20:3n-6) & $0.58 \pm 0.25$ & $0.67 \pm 0.25$ & $0.74 \pm 0.35$ & \\
\hline Arachidonic acid (20:4n-6) & $5.71 \pm 1.10$ & $6.05 \pm 1.08$ & $5.82 \pm 1.03$ & \\
\hline Eicosapentaenoic acid (20:5n-3) & $2.23 \pm 1.02$ & $2.55 \pm 1.44$ & $2.90 \pm 1.54$ & \\
\hline Docosahexaenoic acid $(22: 6 n-3)$ & $1.03 \pm 0.20$ & $1.13 \pm 0.35$ & $1.13 \pm 0.34$ & \\
\hline \multicolumn{5}{|l|}{ Estimated desaturase } \\
\hline D9-16D (16:1n-7/16:0) & $0.19 \pm 0.05$ & $0.22 \pm 0.08$ & $0.29 \pm 0.08^{* \#}$ & $<0.010$ \\
\hline D9-18D (18:1n-9/18:0) & $19.00 \pm 4.04$ & $19.52 \pm 4.06$ & $18.72 \pm 3.66$ & \\
\hline D6D (18:3n-6/18:2n-6) & $0.011 \pm 0.006$ & $0.017 \pm 0.010^{*}$ & $0.018 \pm 0.011^{*}$ & $<0.050$ \\
\hline D5D (20:4n-6/20:3n-6) & $12.27 \pm 3.82$ & $9.53 \pm 2.82^{*}$ & $8.16 \pm 3.20^{*}$ & $<0.001$ \\
\hline \multicolumn{5}{|l|}{ Lipogenic index } \\
\hline $16: 0 / 18: 2 n-6$ & $0.24 \pm 0.05$ & $0.25 \pm 0.03$ & $0.27 \pm 0.04^{*}$ & $<0.050$ \\
\hline
\end{tabular}

Mean \pm SD. ${ }^{*} p<0.05$; versus control group, ${ }^{*} p<0.05$; versus OB group.

tested for significance using a repeated measures oneway ANOVA followed by Tukey's test, or the non-parametric Kruskal-Wallis test followed by the Mann-Whitney's $U$ test adjusted with the Bonfferoni test. Logistic regression analyses were carried out to estimate the risks of abdominal obesity and metabolic syndrome, and a standardized $(\mathrm{SD}=1.0)$ odds ratio $(\mathrm{OR})$ was calculated. Correlation coefficients were determined by single or multiple linear regression analyses. $p<0.05$ was considered to indicate statistical significance.

\section{RESULTS}

Subject characteristics

Clinical characteristics and serum adipocytokine lev- els are presented in Table 1. BMI, waist circumference, triacylgycerol, fasting blood glucose, fasting blood insulin, HbA1c, HOMA-IR, and leptin were significantly higher, and HDL-cholesterol was lower, in the OB group than the control group. In addition to these differences observed in the OB group, the MS group had higher levels of triacylglycerol, fasting blood glucose, and $\mathrm{HbA1c}$ than the OB group. Systolic blood pressure and diastolic blood pressure were significantly higher in the MS group than the control and OB groups.

Fatty acid composition of plasma CEs

The fatty acid composition of plasma CEs is given in Table 2. In the OB group, there was no significant difference in fatty acid composition compared with the con- 
Table 3. Energy, macronutrient and fatty acid intakes.

\begin{tabular}{|c|c|c|c|}
\hline$(/ \mathrm{d})$ & $\begin{array}{c}\text { Control } \\
n=27\end{array}$ & $\begin{array}{c}\text { OB } \\
n=43\end{array}$ & $\begin{array}{c}\text { MS } \\
n=24\end{array}$ \\
\hline Energy (kcal/kg IBW) & $32.34 \pm 4.30$ & $32.34 \pm 6.88$ & $29.51 \pm 5.25$ \\
\hline Protein (g/kg IBW) & $1.07 \pm 0.17$ & $1.07 \pm 0.21$ & $1.04 \pm 0.23$ \\
\hline Carbohydrate (g/kg IBW) & $3.28 \pm 2.04$ & $2.24 \pm 2.02$ & $2.65 \pm 1.77$ \\
\hline Fat (energy\%) & $26.01 \pm 7.04$ & $25.19 \pm 5.79$ & $26.43 \pm 5.39$ \\
\hline \multicolumn{4}{|l|}{ Fatty acids } \\
\hline SFA (g) & $17.22 \pm 5.48$ & $17.47 \pm 5.59$ & $16.48 \pm 5.26$ \\
\hline MUFA (g) & $21.72 \pm 7.93$ & $19.77 \pm 5.63$ & $20.96 \pm 7.53$ \\
\hline PUFA (g) & $13.47 \pm 5.04$ & $11.83 \pm 3.20$ & $11.37 \pm 3.99$ \\
\hline n-3 PUFA (g) & $2.84 \pm 1.42$ & $2.12 \pm 0.59$ & $2.06 \pm 0.91$ \\
\hline n-6 PUFA (g) & $10.55 \pm 4.61$ & $9.78 \pm 2.98$ & $9.08 \pm 3.45$ \\
\hline$n-6 / n-3$ & $4.55 \pm 1.82$ & $4.68 \pm 1.13$ & $4.89 \pm 1.93$ \\
\hline Linoleic acid (g) & $10.29 \pm 4.61$ & $10.25 \pm 2.92$ & $9.71 \pm 4.10$ \\
\hline$\alpha$-Linolenic acid (g) & $1.33 \pm 0.61$ & $1.38 \pm 0.49$ & $1.30 \pm 0.62$ \\
\hline Arachidonic acid (g) & $0.172 \pm 0.098$ & $0.160 \pm 0.082$ & $0.154 \pm 0.060$ \\
\hline Eicosapentaenoic acid (g) & $0.274 \pm 0.258$ & $0.269 \pm 0.211$ & $0.175 \pm 0.126$ \\
\hline Docosahexaenoic acid (g) & $0.093 \pm 0.074$ & $0.082 \pm 0.048$ & $0.079 \pm 0.062$ \\
\hline
\end{tabular}

Mean \pm SD.

No significant difference. IBW, ideal body weight; SFA, saturated fatty acid; MUFA, monounsaturated fatty acid; PUFA, polyunsaturated fatty acid.

Table 4. The effects of the desaturase activities on developing abdominal obesity from control, and metabolic syndrome from abdominal obesity.

\begin{tabular}{lcc}
\hline & $\begin{array}{c}\text { Developing OB } \\
\text { from control } \\
(n=70)\end{array}$ & $\begin{array}{c}\text { Developing MS } \\
\text { from OB } \\
(n=67)\end{array}$ \\
\cline { 2 - 3 } & OR $(95 \% \mathrm{CI})$ & OR $(95 \% \mathrm{CI})$ \\
\hline $\mathrm{D} 9-16 \mathrm{D}^{\mathrm{a}}$ & $1.20(0.69-2.07)$ & $2.44(1.29-4.6)^{*}$ \\
$\mathrm{D} 9-18 \mathrm{D}^{\mathrm{a}}$ & $1.14(0.69-1.88)$ & $0.81(0.48-1.37)$ \\
$\mathrm{D} 6 \mathrm{D}^{\mathrm{a}}$ & $1.56(0.97-2.50)$ & $1.20(0.74-1.97)$ \\
$\mathrm{D} 5 \mathrm{D}^{\mathrm{a}}$ & $0.39(0.20-0.77)^{*}$ & $0.56(0.28-1.14)$ \\
\hline
\end{tabular}

${ }^{*} p<0.05$. OR, odds ratio; CI, confidence interval.

${ }^{a}$ Standardised desaturase ratios were used in the analysis.

trol group. The levels of palmitoleic (16:1n-7), oleic (18:1n-9) and $\gamma$-linolenic (18:3n-6) acids were higher, and the level of linoleic acid (18:2n-6) was lower in the MS group than the control group.

Estimated levels of desaturase activity are also presented in Table 2. Higher levels of D6D activity and lower levels of D5D activity were observed in the OB group. In addition to these differences observed in the OB group, levels of D9-16D activity were also significantly higher in the MS group than the control or OB group. The lipogenic index was significantly higher in the MS group than the control group.

Estimation of energy and nutrient intakes

The energy, macronutrient and fatty acids ingested by the subjects are listed in Table 3. No significant difference was observed in these intakes across the control, OB and MS groups.
Effects of desaturase activities on the development of abdominal obesity and metabolic syndrome

To estimate the risks of developing abdominal obesity from non-obesity (control) and of developing metabolic syndrome from abdominal obesity in relation to the desaturases, logistic regression analysis was carried out and the odds ratio (OR) was calculated. As shown in Table 4, the risk of developing abdominal obesity among non-obese controls increased with a lower level of D5D activity $(\mathrm{OR}=0.39, p<0.01)$. Greater D9-16D activity increased the risk of developing metabolic syndrome from abdominal obesity $(\mathrm{OR}=2.44, p<0.01)$. Thus, for each SD increase in D5D activity, there was a decrease of roughly $60 \%$ in the risk of developing abdominal obesity. For D9-16D activity the situation was opposite, with a 2.4-fold increase in the risk of developing metabolic syndrome.

Relationship between D9-16D and D5D activities, clinical characteristics, and energy and nutrient intakes

To investigate the association of D9-16D and D5D activities with clinical characteristics, and energy and nutrient intakes, single and multiple linear regression analyses were performed. As shown in Table 5, the D5D activity inversely correlated with waist circumference, LDL-cholesterol, blood insulin, HbA1c, HOMA-IR, and leptin level, while it positively correlated with HDL-cholesterol and the intake of $n-3$ PUFA, eicosapentaenoic acid (EPA), and docosahexsaenoic acid (DHA). The influences of these variables on D5D activity were examined further with a multiple linear regression analysis. The D5D activity could be explained by the leptin level and intake of EPA. On the other hand, the D9-16D activity positively related with waist circumference, the serum triacylglycerol level, and the lipogenic index. The multiple linear regression analysis of these 
Table 5. Relationship between estimated D6-19 and D5 desaturase and clinical characteristics, and energy and nutrient intakes.

\begin{tabular}{|c|c|c|c|c|c|c|c|c|}
\hline & \multicolumn{4}{|c|}{ D5 desaturase $(n=70)$} & \multicolumn{4}{|c|}{ D9-16 desaturase $(n=67)$} \\
\hline & \multicolumn{2}{|c|}{ Univariate analysis } & \multicolumn{2}{|c|}{ Multivariate analysis } & \multicolumn{2}{|c|}{ Univariate analysis } & \multicolumn{2}{|c|}{ Multivariate analysis } \\
\hline & $\beta$ & $p$ & $\beta$ & $p$ & $\beta$ & $p$ & $\beta$ & $p$ \\
\hline Waist circumference & -0.35 & 0.005 & & & 0.30 & 0.018 & & \\
\hline Systolic blood pressure & -0.09 & 0.498 & & & 0.20 & 0.118 & & \\
\hline Diastolic blood pressure & -0.03 & 0.831 & & & 0.24 & 0.067 & & \\
\hline Total cholesterol & -0.16 & 0.213 & & & 0.11 & 0.411 & & \\
\hline Triacylglycerol & -0.13 & 0.298 & & & 0.26 & 0.041 & 0.25 & 0.034 \\
\hline HDL-cholesterol & 0.31 & 0.013 & & & 0.17 & 0.199 & & \\
\hline LDL-cholesterol & -0.35 & 0.005 & & & -0.03 & 0.844 & & \\
\hline Fasting blood glucose & -0.14 & 0.286 & & & 0.19 & 0.141 & & \\
\hline Fasting blood insulin & -0.45 & 0.000 & & & 0.18 & 0.171 & & \\
\hline $\mathrm{HbAlc}$ & -0.33 & 0.009 & & & 0.03 & 0.850 & & \\
\hline HOMA-IR & -0.44 & 0.000 & & & 0.20 & 0.117 & & \\
\hline Adiponectin & 0.22 & 0.094 & & & -0.12 & 0.377 & & \\
\hline Leptin & -0.35 & 0.005 & -0.38 & 0.001 & 0.16 & 0.203 & & \\
\hline Lipogenic index (16:0/18:2n-6) & -0.02 & 0.835 & & & 0.31 & 0.003 & 0.26 & 0.017 \\
\hline \multicolumn{9}{|l|}{ Energy and nutrient intakes (/d) } \\
\hline Energy intake (kcal/kg IBW) & -0.16 & 0.236 & & & -0.06 & 0.682 & & \\
\hline Protein $(\mathrm{g} / \mathrm{kg}$ IBW) & 0.01 & 0.566 & & & -0.23 & 0.082 & & \\
\hline Carbohydrate (g/kg IBW) & -0.25 & 0.050 & & & -0.06 & 0.654 & & \\
\hline Fat (energy \%) & 0.25 & 0.052 & & & -0.18 & 0.176 & & \\
\hline SFA $(g)$ & 0.04 & 0.767 & & & 0.73 & 0.711 & & \\
\hline MUFA (g) & 0.13 & 0.339 & & & -0.28 & 0.082 & & \\
\hline PUFA (g) & 0.08 & 0.552 & & & -0.12 & 0.301 & & \\
\hline n-6 PUFA (g) & -0.05 & 0.696 & & & -0.15 & 0.190 & & \\
\hline Linoleic acid (g) & -0.05 & 0.677 & & & -0.13 & 0.204 & & \\
\hline Arachidonic acid (g) & 0.14 & 0.214 & & & 0.16 & 0.500 & & \\
\hline n-3 PUFA (g) & 0.40 & 0.002 & & & -0.21 & 0.120 & & \\
\hline$\alpha$-Linolenic acid (g) & 0.04 & 0.699 & & & -0.19 & 0.119 & & \\
\hline Eicosapentaenoic acid (g) & 0.34 & 0.008 & 0.48 & 0.000 & -0.01 & 0.946 & & \\
\hline Docosahexaenoic acid (g) & 0.33 & 0.018 & & & -0.03 & 0.827 & & \\
\hline
\end{tabular}

$\beta$, correlation coefficient.

factors showed that the D9-16D activity could be explained by the serum triacylglycerol level and lipogenic index.

\section{DISCUSSION}

The present study demonstrated that the MS group, but not $\mathrm{OB}$ group, had higher levels of palmitoleic (16:1n-7), oleic (18:1n-9) and $\gamma$-linolenic (18:3n-6) acids and lower levels of linoleic acid (18:2n-6) in plasma CEs than the control. However, the dietary intake of fatty acids in the MS group did not differ from that in the control or OB group. These results suggest that the differences in plasma fatty acid composition in the MS group probably result from endogenous fatty acid synthesis involving the desaturase.

Higher levels of D6D activity and lower levels of D5D activity were observed in the OB group. The logistic regression analysis showed that the low D5D activity was predictive of abdominal obesity. Warensjö et al. reported that a low level of D5D activity was an early sign of the risk of developing metabolic syndrome, which $20 \mathrm{y}$ later resulted in manifest metabolic syn- drome (14). Metabolic syndrome is preceded by abdominal obesity. The control of D5D activity may prevent metabolic syndrome through the inhibition of abdominal obesity. The multiple linear regression analysis demonstrated that the variance in D5D activity could be explained by the serum leptin level and the intake of EPA. It was reported that n-3 PUFAs, EPA and DHA, have beneficial effects on preventing and/or improving obesity (25). Nakatani et al. (26) reported that a highfish-oil intake decreased body weight and fat mass. It is well established that increasing the dietary intake of n-3 PUFAs improves several cardiovascular risk factors, such as hypertension, insulin resistance, and blood lipid levels (27). The intake of $n-3$ PUFAs was also shown to decrease serum leptin levels in humans (28). The intakes of $n-3$ PUFAs such as EPA and DHA promoted the $\beta$-oxidation of fatty acids with a reduction in triacylglycerol biosynthesis in the liver (29). These results suggest that a high intake of EPA reduces fat mass and body weight with increasing D5D activity. The intake of EPA would have the beneficial effects on the prevention and improvement of abdominal obesity. 
The level of D9-16D activity was higher in the MS group than the control and OB groups. The logistic regression analysis showed that the high level of D9$16 \mathrm{D}$ activity was predictive of the development of metabolic syndrome from abdominal obesity. In the multiple linear regression analysis, D9-16D activity was related to the serum triacylglycerol level and lipogenic index. Attie et al. (16) and Zhou et al. (30) also reported that D9D activity was correlated with serum triacylglycerol levels in human subjects. Stearoyl-CoA desaturase 1 (alternatively known as D9D) has been shown to be a rate-limiting enzyme for the synthesis of triglycerides by Miyazaki et al. (31). The intake of SFA or carbohydrates, which elevate the serum triacylglycerol level, has been suggested to elevate the D9D level (32). However, D9$16 \mathrm{D}$ activity was not significantly related to dietary intake of SFA and carbohydrate in this study. Enhanced endogenous lipogenesis would explain the higher levels of triacylglycerol in serum and subsequent D9D activity. In fact, a higher lipogenic index was observed and positively correlated with D9-16D activity in the MS group. Suppression of lipogenesis and a subsequent decrease in the serum level of triacylglycerol may prevent the development of metabolic syndrome from abdominal obesity through the regulation of D9D activity.

In conclusion, our results suggest that estimated D5D and D9-16D activities can be used to predict abdominal obesity and metabolic syndrome. The risk of developing abdominal obesity dropped by $60 \%$ for each SD increase in D5D activity, and the risk of developing metabolic syndrome from abdominal obesity increased 2.4 times for each SD increase in D9-16D activity. In the multiple linear regression models, D5D and D9-16D positively correlated with the intake of EPA and lipogenic index, respectively. These suggest that the intake of EPA is beneficial to prevent abdominal obesity and the development of metabolic syndrome.

\section{Acknowledgments}

This study was supported by Grants-in-aid for Scientific Research from the Ministry of Education, Culture, Sports, Science and Technology of Japan, and by grants for project research from Nara Women's University.

\section{REFERENCES}

1) Grundy SM, Brewer HB, Cleeman JI, Smith SC, Lenfant C. 2004. Definition of metabolic syndrome. Report of the National Heart, Lung, and Blood Institute/American Heart Association conference on scientific issues related to definition. Arterioscler Thromb Vasc Biol 24: 13-18.

2) Isomaa B, Almgren P, Tuomi T, Forsén B, Lahti K, Nissén M, Taskinen MR, Groop L. 2001. Cardiovascular morbidity and mortality associated with the metabolic syndrome. Diabetes Care 24: 683-689.

3) Li JJ, Huang CJ, Xie D. 2008. Anti-obesity effects of conjugated linoleic acid, docosahexaenoic acid, and eicosapentaenoic acid. Mol Nutr Food Res 52: 631-645.

4) Lovejoy J, DiGirolamo M. 1992. Habitual dietary intake and insulin sensitivity in lean and obese adults. Am J Clin Nutr 55: 1174-1179.
5) Parks EJ, Hellerstein MK. 2000. Carbohydrate-induced hypertriacylglycerolemia: historical perspective and review of biological mechanisms. Am J Clin Nutr 71: 412-433.

6) Katan MB, Deslypere JP, van Birgelen AP, Penders M, Zegwaard M. 1997. Kinetics of the incorporation of dietary fatty acids into serum cholesteryl esters, erythrocyte membranes, and adipose tissue: an 18-month controlled study. J Lipid Res 38: 2012-2022.

7) Dougherty RM, Galli C, Ferro-Luzzi A, Iacono JM. 1987. Lipid and phospholipid fatty acid composition of plasma, red blood cells, and platelets and how they are affected by dietary lipids: a study of normal subjects from Italy, Finland, and the USA. Am J Clin Nutr 45: 443-455.

8) Ma J, Folsom AR, Shahar E, Eckfeldt JH. 1995. Plasma fatty acid composition as an indicator of habitual dietary fat intake in middle-aged adults. The Atherosclerosis Risk in Communities (ARIC) Study Investigators. Am J Clin Nutr 62: 564-571.

9) Vessby B, Gustafsson IB, Tengblad S, Boberg M, Andersson A. 2002. Desaturation and elongation of fatty acids and insulin action. Ann NY Acad Sci 967: 183-195.

10) Wang L, Folsom AR, Zheng ZJ, Pankow JS, Eckfeldt JH; ARIC Study Investigators. 2003. Plasma fatty acid composition and incidence of diabetes in middle-aged adults: the Atherosclerosis Risk in Communities (ARIC) Study. Am J Clin Nutr 78: 91-98.

11) Laaksonen DE, Lakka TA, Lakka HM, Nyyssönen K, Rissanen T, Niskanen LK, Salonen JT. 2002. Serum fatty acid composition predicts development of impaired fasting glycaemia and diabetes in middle-aged men. Diabet Med 19: 456-464.

12) Vessby B. 2003. Dietary fat, fatty acid composition in plasma and the metabolic syndrome. Curr Opin Lipidol 14: $15-19$.

13) Warensjö E, Ohrvall M, Vessby B. 2006. Fatty acid composition and estimated desaturase activities are associated with obesity and lifestyle variables in men and women. Nutr Metab Cardiovasc Dis 16: 128-136.

14) Warensjö E, Risérus U, Vessby B. 2005. Fatty acid composition of serum lipids predicts the development of the metabolic syndrome in men. Diabetologia 48: 19992005.

15) Maruyama C, Yoneyama M, Suyama N, Yoshimi K, Teramoto A, Sakaki Y, Suto Y, Takahashi K, Araki R, Ishizaka Y, Yamakado M, Teramoto T. 2008. Differences in serum phospholipid fatty acid compositions and estimated desaturase activities between Japanese men with and without metabolic syndrome. J Atheroscler Thromb 15: 306-313.

16) Attie AD, Krauss RM, Gray-Keller MP, Brownlie A, Miyazaki M, Kastelein JJ, Lusis AJ, Stalenhoef AF, Stoehr JP, Hayden MR, Ntambi JM. 2002. Relationship between stearoyl-CoA desaturase activity and plasma triglycerides in human and mouse hypertriglyceridemia. J Lipid Res 43: 1899-1907.

17) The Examination Committee of Criteria for Metabolic Syndrome. 2005. Definition and criteria for metabolic syndrome. J Jpn Soc Int Med 94: 794-809.

18) Satoh N, Ogawa Y, Usui T, Tagami T, Kono S, Uesugi H, Sugiyama H, Sugawara A, Yamada K, Shimatsu A, Kuzuya H, Nakao K. 2003. Antiatherogenic effect of pioglitazone in type 2 diabetic patients irrespective of the responsiveness to its antidiabetic effect. Diabetes Care 26: 2493-2499. 
19) Bligh EG, Dyer WJ. 1959. A rapid method of total lipid extraction and purification. Can J Biochem Physiol 37: 911.

20) Okita M, Hayashi M, Sasagawa T, Takagi K, Suzuki K, Kinoyama S, Ito T, Yamada G. 2001. Effect of a moderately energy-restricted diet on obese patients with fatty liver. Nutrition 17: 542-547.

21) Hudgins LC, Hellerstein M, Seidman C, Neese R, Diakun J, Hirsch J. 1996. Human fatty acid synthesis is stimulated by a eucaloric low fat, high carbohydrate diet. $J$ Clin Invest 97: 2081-2091.

22) Miyaki K, Sutani S, Kikuchi H, Takei I, Murata M, Watanabe K, Omae K. 2005. Increased risk of obesity resulting from the interaction between high energy intake and the Trp64Arg polymorphism of the beta3adrenergic receptor gene in healthy Japanese men. J Epidemiol 15: 203-210.

23) Science and Technology Agency. 2005. Standard Tables of Food Composition in Japan, Fatty Acid Section, 5th revised and enlarged ed, Printing Bureau of the Ministry of Finance, Tokyo.

24) Suzuki K, Sasagawa T, Okita O, Fujiwara Y, Mori K, Saito M, Abe Y. 1996. Development of new simplified-food frequency method for use in the surveillance on life style in order to promote healthy condition in Okayama Prefecture, computed by Microsoft Excel. Bull Fac Health Welfare Sci Okayama Prefect Univ 3: 143-153.

25) Li JJ, Huang CJ, Xie D. 2008. Anti-obesity effects of conjugated linoleic acid, docosahexaenoic acid, and eicosapentaenoic acid. Mol Nutr Food Res 52: 631-645.

26) Nakatani T, Kim HJ, Kaburagi Y, Yasuda K, Ezaki O.
2003. A low fish oil inhibits SREBP-1 proteolytic cascade, while a high-fish-oil feeding decreases SREBP-1 mRNA in mice liver: relationship to anti-obesity. J Lipid Res 44: 369-379.

27) Robinson LE, Buchholz AC, Mazurak VC. 2007. Inflammation, obesity, and fatty acid metabolism: influence of n-3 polyunsaturated fatty acids on factors contributing to metabolic syndrome. Appl Physiol Nutr Metab 32: 1008-1024.

28) Ramel A, Parra D, Martinéz JA, Kiely M, Thorsdottir I. 2009. Effects of seafood consumption and weight loss on fasting leptin and ghrelin concentrations in overweight and obese European young adults. Eur J Nutr 13 [PMID: 19142567].

29) Jacobson TA. 2008. Role of $n-3$ fatty acids in the treatment of hypertriglyceridemia and cardiovascular disease. Am J Clin Nutr 87: 1981S-1990S.

30) Zhou YE, Egeland GM, Meltzer SJ, Kubow S. 2009. The association of desaturase 9 and plasma fatty acid composition with insulin resistance-associated factors in female adolescents. Metabolism 58: 158-166.

31) Miyazaki M, Kim YC, Gray-Keller MP, Attie AD, Ntambi JM. 2000. The biosynthesis of hepatic cholesterol esters and triglycerides is impaired in mice with a disruption of the gene for stearoyl-CoA desaturase 1. J Biol Chem 275: 30132-30138.

32) Warensjö E, Risérus U, Gustafsson IB, Mohsen R, Cederholm T, Vessby B. 2008. Effects of saturated and unsaturated fatty acids on estimated desaturase activities during a controlled dietary intervention. Nutr Metab Cardiovasc Dis 18: 683-690. 